\title{
La cuestión «racial» en Cuba actual: algunas consideraciones
}

\section{Jesús Guanche Pérez}

Centro de Investigación y D esarrollo de la M úsica Cubana (CID M UC).

Calle G, núm. 505/21 y 23. 10400 Velado. La H abana. Cuba

Fecha de recepción: 1996

\section{Resumen}

El presente trabajo valora cuestiones principales sobre la racialidad - en este caso como construcción cultural- en la sociedad cubana de los años noventa. La presencia del prejuicio racial en las estructuras horizontales de la sociedad, no obstante las acciones para transformar estas diferencias en el orden legal. Considera el papel de la autoimagen racial vinculada con la pertenencia social y su reproducción desde el nivel personal y familiar.

Palabras clave: autoimagen racial, fenotipos populares, racialidad, endoculturación familiar.

Abstract. The «racial» problem in present Cuba: some considerations

The present paper rate the essential problems about raciality - in this case as cultural construction - in the cuban society at the ninety years. The presence of racial prejudice in horizontal structure of the society, however the actions to transform that differences in legal regime. Conside the rol of racial selfimage in relation with the social position and his reproduction from personal and familiar level.

Key words: racial sefimage, popular fenotipics, raciality, domestic endoculturation.

\section{Sumario}

Introducción Confusión de lo «racial» por lo «étnico»

El hábil engaño de las «razas» La autoimagen racial del cubano 


\section{Introducción}

L os estudios sobre relaciones raciales en Cuba cuentan con una abundante bibliografía que permite conocer el desarrollo histórico de esta temática, los vínculos biológicos y culturales entre diferentes grupos humanos procedentes de diversas latitudes; así como la intensa y creciente mezcla que se efectúa entre la propia población nacida y multiplicada durante decenas de generaciones en la isla, independientemente de sus características morfológicas externas ${ }^{1}$.

N o obstante los esfuerzos realizados por cientos de antropólogos físicos y socioculturales de todo el orbe, todavía se confunde - tanto en los medios de comunicación masiva de Cuba como a nivel de las instituciones del Estadola sustancial diferencia entre lo étnico y lo racial 2 .

D e manera sintética, las diferencias esenciales entre lo étnico y lo racial están dadas por las cual idades específicas de la cultura y la natura, respectivamente; pero esta definición simple no es una camisa de fuerza, ya que también se efectúa una permanente interacción entre las características culturales de cualquier etnos y las capacidades de adaptabilidad y mutabilidad de las personas que a él pertenecen.

El presente trabajo tiene como principal propósito valorar cuestiones generales de la racialidad - en este caso como construcción cultural - en la sociedad cubana de los años noventa.

\section{El hábil engaño de las «razas»}

La racialidad es una noción que ha conducido a engaño, por el lastre conceptual y activo del racismo y los prejuicios raciales, ya que lejos de valorar lo estrictamente biológico, posee una marcada connotación sociocultural y clasista. Por ello, determinados autores prefieren hablar de «raza social» ${ }^{3}$ y asumirlo como una construcción cultural ${ }^{4}$ debido a sus múltiples implicaciones en las relaciones sociales.

En este sentido, Cuba heredó un profundo estigma en la discriminación y los prejuicios raciales, derivados de la etapa colonial por el impacto, tanto de la esclavitud de los africanos y sus descendientes, como en las relaciones globales de dominación respecto del grueso de la población más humilde - con inde-

1. Véanse Fernán dez Ro bain A, Tomás (1985). Bibliografía de temas afrocubanos. Biblioteca Nacional José Martí, La Habana; y del propio autor: (1994). Cultura afrocubana. Cuadernos de Bibliografía Cubana, núm. 1. Biblioteca N acional José M artí, La H abana.

2. Estos términos son usados indistintamente por autores de diversos países; véanse de BRETO n, Roland J.L. (1983). Las etnias. Barcelona; y de BRo m LEI, Yu. (1986). «Etnos y sus tipos principales», en Etnografía teórica. M oscú, p. 6-38; así como sus respectivas bibliografías.

3. Véase W AGLEY, Charles (1968). «T he Concept of Social Race in the Americas», en The Latin American Tradicion. N ueva York, p. 155-174.

4. Véase KотTAK, Conrad Phillip (1994). «La construcción cultural de la raza», en Antropología. Una exploración de la diversidad humana con temas de la cultura hispana. M adrid, p. 79-94. 
pendencia de sus rasgos externos- , principalmente debido a sus condiciones sociales.

La etapa neocolonial también acrecentó el racismo estamentador de grupos sociales según la cantidad de melanina en la piel, lo que representó una forma externa de influir negativamente en la unidad nacional, a la vez que generó un fuerte movimiento social antirracista de proyección democráticopopular, liderado por destacados intelectuales con el apoyo del movimiento obrero y estudiantil.

Tras la lucha de liberación en 1959 una parte del programa de transformaciones estuvo encaminado a erradicar este enraizado lastre social. Se pensó ingenuamente que si se eliminaban las vías institucionales que propiciaban la práctica de la discriminación racial y se enfatizaba en la educación y en la convivencia cotidiana, automáticamente se podían barrer las raíces del racismo y de los prejuicios raciales. En este sentido, al gunos autores llegaron a afirmar categóricamente que el problema había sido resuel to y que ya era cosa del pasado 5 .

En el capítulo V de la Constitución de la República de Cuba, dedicado a la I gualdad, aprobada el 24 de febrero de 1976, se señala en su artículo 41 que: «La discriminación por motivo de raza, color, sexo u origen nacional está proscrita y sancionada por la ley. Las instituciones del Estado educan a todos, desde la más temprana edad, en el principio de la igualdad de los seres humanos» ${ }^{\circ}$.

Esta Constitución, que fue modificada en 1992 con el objetivo de adecuarla a las nuevas condiciones históricas, entre ellas, al crecimiento de las inversiones extranjeras y sus alcances jurídicos, no alteró dicho principio.

Efectivamente, la apertura del acceso de la población epitelialmente clasificada de «negra» y «mulata» a los lugares públicos que les eran vedados (playas, casinos, clubes, hoteles y otros), la posibilidad de acceder libre y gratuitamente a la enseñanza en todos los niveles, a los servicios de salud, deportivos y culturales, así como a diversos puestos de trabajo y cargos de dirección, contribuyeron a romper una primera barrera, la del nivel vertical (institucional); pero dejaba sin revolucionar otra más profunda y diversa, que se reproduce y se multiplica a nivel horizontal; desde la autoestima personal, los complejos psicológicos heredados y transmitidos, que condicionan la autoimagen sobre la supuesta «pertenencia racial», Ios gustos estéticos para la elec-

5. Véanse CARNEADO, José F. «La discriminación racial en Cuba no volverá jamás», en rev. Cuba socialista, año 2, núm. 5. La H abana, enero de 1962, p. 54-67; Serviat, Pedro «a discriminación racial en Cuba, su origen, desarrollo y terminación definitiva», en rev. Isas, núm. 66, Santa Clara, mayo-agosto de 1980, p. 3-22; FERRER, Rodolfo M. «El problema negro en Cuba y su solución definitiva», en El militante comunista, La H abana, octubre de 1986, p. 65-66; y SeRviat, Pedro. (1986). El problema racial en Cuba y su solución definitiva, La H abana: Editora Política.

6. Constitución de la República de Cuba. La H abana, 1976, p. 33. 
ción de pareja, los vínculos de la pareja antes y después del matrimonio, las relaciones familiares y vecinales, así como entre los diversos grupos socioocupacionales, entre muchos aspectos.

\section{Los fenotipos populares}

Aunque varios autores afirman con razón que la clasificación racial está fuera de lugar en biología ${ }^{7}$, debido a los múltiples problemas para agrupar a pueblos espećficicos en «hipotéticas» unidades raciales aisladas y distintas; tal como señala Wenda Trevathan: «Evitar la raza, tratarla como si no existiese como concepto, válido o no, en antropología física, es adoptar la posición del avestruz en el mejor de los casos, una posición no ética en el peor» ${ }^{8}$. En algunos países como Brasil las formas de denominar los tipos humanos o fenotipos son menos excluyentes y flexibles, la noción de «raza» puede cambiarse con la mejoría del status social; y determinados autores han compilado más de 500 «etiquetas raciales»? 9 .

En Cuba también existen clasificaciones «raciales» de tipo popular, que prestan atención - como en otros países de América Latina y el Caribe hispanohablante - a las características evidentes del color de la piel, la forma y el color del cabello y el color de los ojos. De las denominaciones populares recogidas a manera de ejemplos, sólo una incluye, como veremos, la forma del cráneo.

Si ordenamos los fenotipos populares cubanos según la intensidad de la coloración epitelial, éstos son:

1. negro-azul: piel muy morena y mate; pelo muy rizado y negro; ojos negros;

2. negro color teléfono: piel muy morena y brillosa; pelo muy rizado y negro; ojos negros;

3. negro coco timba: piel morena o muy morena; pelo muy rizado, negro, en forma de granos de pimienta y separados entre sí; ojos negros;

4. negro cabeza de puntilla: piel morena o muy morena, pelo muy rizado y negro; ojos negros y prominente dolicocefalia;

5. negro: piel morena de diversos matices; pelo muy rizado y negro; ojos negros o castaño oscuro;

6. moro: piel morena, pelo poco rizado y negro; ojos negros;

7. mulato: piel canela de variada intensidad; pelo rizado y negro; ojos castaño oscuros o negros;

8. indio: piel canela o bronceada; pelo lacio muy negro y brillante; ojos negros y con frecuencia rasgados por el pliegue epicántico;

7. Véanse $O$ RTIZ, Fernando (1946). El engaño de las razas, La Habana; también H ARRIS, M arvin (1974). (O p. cit., p. 121) hace referencia a los trabajos de M ontagu; Fried, 1968; Littlefield, Lieberman, Reynolds, 1982.

8. H arris cit. Trevethan. Op. cit., p. 121.

9. Véase H ARRIS, M arvin (1970). «R eferential Ambiguity in the Calculus of Braziliam Racial Identity». Sonthmestern Journal at Anthropology, 26, p. 1-9. 
9. mulato chino: piel canela o canela clara; pelo algo rizado; ojos negros rasgados por el pliegue epicántico;

10. mulato color cartucho: piel canela clara; pelo ligeramente rizado y castaño oscuro o negro; ojos castaño oscuros o negros;

12. trigueño: piel bronceada; pelo algo rizado y negro; ojos negros;

13. jabao: piel canela clara u ocre; pelo rizado y amarillo oscuro; ojos castaño claros o verde claros;

14. colorao: piel rojiza y regularmente pecosa; pelo rizado u ondulado y rojizo; ojos castaños o castaño claros;

15. chino: piel clara amarillenta; pelo muy lacio y negro; ojos negros y rasgados por el pliegue epicántico;

16. blanco: piel clara, pelo lacio u ondulado y castaño o negro; ojos castaños o negros;

17. rubi o: piel clara; pelo lacio u ondulado y amarillo claro u oscuro; ojos verdes, azules o castaño claros;

18. blanco orillero: puede tener una aceptación social como sinónimo de marginal o una acepción biológica como sinónimo de mezcla racial; posee piel clara pero muy resistente al sol del trópico; pelo ondulado o rizado y negro; ojos castaño oscuros o negros;

19. blanco lechoso: piel muy clara y regularmente pecosa; pelo lacio u ondulado, castaño claro; ojos castaños o negros;

20. albino: piel despigmentada; pelo rizado o muy rizado y amarillo claro; ojos claros.

Todas estas denominaciones pueden tener, de acuerdo con el contexto, una connotación afectiva o despectiva. Un término muy usado como «mi negro(a)» puede ser sinónimo de «mi niño(a)»o de «mi socio(a)» y emplearse para designar personas de las más variadas pigmentaciones. D e igual manera, el uso de diminutivos (ito-ita/ico-ica) sirven para suavizar las denominaciones interpersonales con una implicación afectiva o simplemente indicativa. M uchos prefieren decir «negrito(a)», «prietecito(a)», «mulatico(a)», «blanquito(a)» y no emplean el sustantivo como tal por la histórica implicación despectiva 0 de dominación que ha tenido o que aún tiene en determinados medios familiares y sociales. En este caso, los diminutivos se emplean independientemente de la edad de las personas a las que se refieren.

Aunque los matrimonios epitelialmente mixtos y estables han tendido a crecer en los últimos treinta y cinco años, sean estos consensuales o legitimados por la ley o ante determinado credo religioso, todavía se observan criterios propios del lenguaje popular que trascienden al nivel intergeneracional (lo que se conoce técnicamente como la endoculturación) sobre la aspiración de una joven de piel morena y pelo rizado de tener un hijo de piel clara y pelo lacio con el objetivo de «mejorar la raza», como evidente reflejo de los prejuicios raciales respecto de sí misma. En el sentido estrictamente biológico, este criterio pudiera ser válido si la joven viviera en un país nórdico; pero en el trópico, la piel morena y el pelo rizado son, sin duda, mucho mejores para resistir los rayos solares, evitar el cáncer de piel y propiciar una mejor transpiración. 
Tabla 1. Composición de la población de Cuba según el color de la piel, por sexos (1981).

\begin{tabular}{lrrrrrr}
\hline Color de la piel & $\begin{array}{l}\text { Varones } \\
\text { (en miles) }\end{array}$ & $\%$ & $\begin{array}{l}\text { H embras } \\
\text { (en miles) }\end{array}$ & $\%$ & $\begin{array}{l}\text { Total } \\
\text { (en miles) }\end{array}$ & $\%$ \\
\hline Blancos & $3.239,8$ & 65,9 & $3.175,7$ & 66 & $6.415,5$ & 66 \\
N egros & 603,3 & 12,3 & 565,4 & 11,8 & $1.168,7$ & 12 \\
Asiáticos & 8,4 & 0,2 & 5,6 & 0,1 & 14 & 0,1 \\
M estizos & $1.063,4$ & 21,6 & 1.062 & 22,1 & $2.125,4$ & 21,9 \\
Total & $4.914,9$ & & $4.808,7$ & & $9.723,6$ & \\
\hline
\end{tabular}

Fuente: Com Ité Estatal de Estadísticas. O ficina Nacional del Censo. Censo de Población y Viviendas de 1981, tomo XVI.

\section{Confusión de «racial» por lo «étnico»}

A mediados de la década de los ochenta, debido al indiscutible peso de la participación femenina ${ }^{10}$ y de la población más joven en el desarrollo socioeconómico del país y la necesidad de su promoción y apoyo, también se introdujo el tema de una falsa «composición étnica» limitada a lo epitelial, como otro posible indicador que, lejos de contribuir a resolver la raíz sociocultural de este problema, volvió a sacar a la superficie una cuestión más compleja cuya solución depende del desarrollo socioeconómico sostenible y de una mejor equidad en cuanto a derechos y deberes de cada uno de los miembros de la sociedad. Esta concepción estuvo cargada, en esa ocasión, de un sutil paternalismo con nuevos matices de discriminación en el sentido positivo. En el Informe Central al Tercer Congreso del Partido Comunista de Cuba se señalaba: «H ay que asegurar una adecuada representación femenina acorde con la participación y el aporte importante de las mujeres [... ] y la presencia de la creciente cantera de jóvenes y prometedores valores nacidos y forjados en la Revolución. La composición étnica de nuestro pueblo, unida al mérito revolucionario y al talento probado de muchos compatriotas, que en el pasado eran discriminados por el color de la piel, debe estar justamente representada en los cuadros dirigentes del Partido» ${ }^{11}$.

En realidad no se tomaba en consideración la verdadera composición étnica, sino sólo la composición según la cantidad de melanina, el indicador más evidente y superficial en las diferencias biológicas de los seres humanos, para tratar de resolver un problema económico y sociocultural.

Para ello se basaron en los resultados del censo de 1981 que - a diferencia de las múltiples denominaciones fenotípicas populares señaladas- sólo incluyó la habitual clasificación de «blancos, negros, asiáticos y mestizos» (tabla 1).

La información obtenida fue de hecho falsa por diversas razones: el instrumento de observación - la encuesta nacional - autolimitaba los índices a

10. En ese momento las mujeres constituían el $37,3 \%$ de la fuerza laboral activa en el sector estatal civil y el $55,4 \%$ de la fuerza técnica (profesionales de nivel medio y superior), con evidente tendencia a crecer en cerca del $1 \%$ anual.

11. La H abana, 1986, p. 95-96. 
Tabla 2. Composición de la población de Cuba según el color de la piel, por provincias, en \% (1981)

\begin{tabular}{lllll}
\hline Provincias & Blancos & Negros & Asiáticos & M estizos \\
\hline Pinar del Río & 78,3 & 14,3 & 0 & 7,4 \\
La H abana & 82,2 & 9,5 & 0,1 & 8,2 \\
C. de La H abana & 63 & 16,4 & 0,2 & 20,4 \\
M atanzas & 76 & 12,7 & 0,1 & 11,2 \\
Villa Clara & 82,5 & 6,9 & 0,1 & 10,5 \\
Cienfuegos & 76,6 & 9,6 & 0,1 & 13,7 \\
Sancti Spiritus & 84,1 & 7,4 & 0,0 & 8,5 \\
Ciego de Ávila & 80,8 & 9,5 & 0,1 & 9,6 \\
Camagüey & 77 & 11 & 0,1 & 11,9 \\
Las Tunas & 74,4 & 7,2 & 0,1 & 18,3 \\
H olguín & 78,8 & 6,1 & 0,2 & 14,9 \\
Granma & 42,7 & 4,4 & 0,2 & 52,7 \\
Santiago de Cuba & 30,2 & 22,2 & 0,3 & 47,3 \\
Guantánamo & 26,3 & 18,8 & 0,4 & 54,5 \\
Isla de la Juventud & 66,8 & 10,9 & 0,2 & 22,1 \\
Cuba & 66 & 12 & 0,1 & 21,9 \\
\hline
\end{tabular}

Fuente: Diario Granma, lunes 18 de septiembre de 1983, p. 5.

clasificaciones epiteliales, así como la congruencia taxonómica de sus denominaciones, ya que los de piel amarilla y sus tonalidades no eran necesariamente «asiáticos», dos denominaciones aludían al color («blanco» 0 «negro»), una a un continente («asiáticos»), lo que introduce un criterio geográfico, y otra («mestizos») a la mezcla de los anteriores, pero sin color ni referencia geográfica determinada; los entrevistadores o aplicadores de la encuesta no tenían una preparación en antropología física como para discernir entre unos y otros fenotipos; y la clasificación de los fenotipos dependía de la autoimagen del entrevistado, de modo que en un mismo núcleo familiar podía haber «blancos, mestizos y negros»s según la consideración de cada quien.

Según se puede observar en la tabla 2, quizá uno de los casos más simpáticos es el de la provincia de G uantánamo, donde aparece nada menos que el $0,4 \%$ de población asiática, cuando históricamente esa es un área del país casi no poblada por chinos ni descendientes. Como puede observarse, es la mayor cifra relativa de todo el país. Los que hemos estudiado la historia étnica de Cuba sabemos bien que el grueso del poblamiento chino se asentó en las provincias occidentales (más del $80 \%$ ), sobre todo en La H abana y M atanzas. La presencia china propiamente dicha en la antigua provincia de 0 riente, según el censo de 1970, fue de 15,92\% respecto del resto de la isla. Sin embargo, como los entrevistados observaron una relativa profusión del pliegue epicántico en la población cubana de esa parte del país, muchos de ellos descendientes de los antiguos aruacos y sus disímiles mezclas, y como no tenían otra clasificación posible - que la población denomina «indio» y «mulato chino» , no les quedó otra alternativa que registrarlos como si fueran «asiáticos». 


\section{La autoimagen racial del cubano}

Lo que sí permitió medir el censo desde el punto de vista estadístico, según puede compararse en la tabla 3, es el sentido subjetivo de la autoimagen del cubano. La tendencia al reconocimiento del mestizaje, al hecho de considerarse «mulato» sin una connotación despectiva (no «mestizo» como aparece en los datos - aunque sumamente baja respecto de la realidad- ), conocer el proceso de decrecimiento de la autoimagen del «blanco»y del «negro», respecto de los censos de la primera mitad del presente siglo.

Los esfuerzos realizados en sólo algo más de tres décadas no pueden ser suficientes para superar más de cuatro siglos de dependencia estructural y mental. Las diversas vías de participación sociocultural de la población tampoco pueden medirse por el esquema rígido y prejuiciado de la coloración epitelial en un país donde predominan las mezclas crecientes de toda índole.

Es un hecho evidente que la inmensa mayoría de los equipos deportivos nacionales de al to rendimiento están compuestos por jóvenes «negros» y «mulatos» - según la clasificación popular - , debido al fuerte nivel de prioridad y apoyo estatal que se le ha dado al deporte organizado y de competitividad internacional, que tiene su «secreto» o su «milagro» en la masividad; lo mismo sucede con las agrupaciones de la música popular profesional, en las que muchos de sus integrantes ya son graduados de nivel medio y superior, lo que diferencia sustancialmente esta producción e interpretación musical de la que se efectúa en otros países del Ilamado «Tercer M undo», en cuanto a la calidad y preparación técnica. Los éxitos de las actividades deportivas y artísticas forman parte sustancial del orgullo nacional; pero, al mismo tiempo, la mayor parte de la población reclusa por delitos comunes son también «negros» y «mulatos» y, de ellos, la gran mayoría (más del 90\%) son menores de treinta y cinco años.

En todo este proceso hay que considerar en su justa medida el decisivo papel de la endoculturación familiar: en la transmisión de ejemplos y valores morales y laborales heredados y adquiridos en condiciones históricas de pobreza; el no aprovechamiento óptimo de las oportunidades de estudiar y trabajar; así como el verdadero papel educativo de la escuela, mucho más complejo e integral que el formalmente instructivo.

Tabla 3. Composición de la población de Cuba según el color dela piel (1931-1981)*

\begin{tabular}{lcccc}
\hline Color de la piel & $\mathbf{1 9 3 1}$ & $\mathbf{1 9 4 3}$ & $\mathbf{1 9 5 3}$ & $\mathbf{1 9 8 1}$ \\
\hline Blancos & 72,1 & 74,3 & 72,8 & 66 \\
N egros & 11 & 9,7 & 12,4 & 12 \\
Asiáticos & 0,7 & 0,4 & 0,3 & 0,1 \\
M estizos & 16,2 & 15,6 & 14,5 & 21,9 \\
\hline
\end{tabular}

* El censo de 1970 no recogió este dato.

Fuente: Com ITÉ ESTATAL DE EsTADísticAs. O ficina Nacional del Censo. Censo de Población y Viviendas de 1981, tomo XVI. 
En el caso de la población «negra»y «mulata» no es lo mismo la parte descendiente de hombres y mujeres libres por varias generaciones, portadora de una rica tradición laboral, poseedora de los principales oficios y algunas profesiones de prestigi $0^{12}$; que la parte descendiente de la población esclava, hace sólo tres o cuatro generaciones, quienes han padecido el desempleo y el subempleo crónicos, que han vivido en condiciones de promiscuidad y hacinamiento $y$ en la que se ha enraizado una marginalidad no sólo espacial - en cuanto asentamiento habitacional - sino también psicológica.

En el caso de la población autodenominada «blanca», tampoco es lo mismo la parte descendiente por muchas generaciones de pobladores urbanos y rurales con recursos económicos y con posibilidades de abrirse paso en la sociedad, que la parte descendiente - en su inmensa mayoría- de hombres y mujeres humildes, que han constituido el proletariado, el campesinado y otros grupos y capas sociales, muchos de los cuales también han vivido en condiciones infrahumanas.

La base real del problema, para glosar términos anatómicos, no es epitelial, es decir, «superficial», sino medular, o sea, «mucho más profunda». Se encuentra en la conocida división de la sociedad en clases, grupos y capas, en las relaciones de propiedad, generadoras de múltiples nexos sociales, condicionadora de la estructura y la jerarquía familiar, en la propia psicología individual y social, en las posibilidades del desarrollo pleno de las capacidades y en la diversificación de aspiraciones y oportunidades.

Los recientes estudios sobre Relaci ones raciales y etnicidad en la sociedad cubana actual, iniciados por el Centro de Antropología del M inisterio de Ciencia, Tecnología y M edioambiente, deben aportar nuevos resultados a partir del trabajo de campo y del debate científico abierto, que permita una evaluación más profunda y crítica sobre una problemática contemporánea que forma parte indisoluble de la cuestión nacional.

Esta reflexión y acción colectiva también permitirá compartir experiencias con otros países que han sido afectados nuevamente por brotes de racismo, prejuicios raciales, xenofobia y diversos estallidos sociales, así como la peculiar etnofobia intraétnica vs xenofilia interétnica a partir del impacto del turismo internacional y la irrupción del capital extranjero, que se traduce como apertura exterior y apretura interior.

El carácter uniétnico y multirracial de la nación cubana, en tanto construcción cultural diversa, representa una cualidad histórica válida para el conocimiento de otros pueblos del área y del orbe.

12. Véase D escham Ps Chapeaux, Pedro (1970). El negro en la economía habanera del siglo xix. La H abana. 